\section{Morphological changes in the fast vs slow fiber profiles of the urethras of diabetic pregnant rats}

\author{
Gabriela Marini, ${ }^{1}$ Angélica M. Pascon \\ Barbosa, ${ }^{1}$ Débora C. Damasceno, \\ Selma M. Michelin Matheus, ${ }^{2}$ \\ Rodrigo de Aquino Castro, ${ }^{3}$ \\ Manoel João B. Castello Girão, ${ }^{3}$ \\ Marilza V. Cunha Rudge ${ }^{1}$ \\ 'Experimental Research Laboratory, \\ Department of Gynecology and \\ Obstetrics, Botucatu Medical School, \\ UNESP - Univ. Estadual Paulista, São \\ Paulo; 'Department of Anatomy, \\ Botucatu Biosciences Institute, UNESP - \\ Univ. Estadual Paulista, São Paulo; \\ ${ }^{3}$ Department of Gynecology, São Paulo \\ Federal University (UNIFESP), São Paulo, \\ Brazil
}

\section{Abstract}

Background. This study was undertaken to test the hypothesis that diabetes and pregnancy detrimentally affect the normal function of urethral striated muscles in rats, providing a model for additional studies related to urinary incontinence. The aim of this study was to evaluate morphological alterations in the urethral striated muscles of diabetic pregnant rats.

Materials and Methods. Twenty female Wistar rats were distributed into four experimental groups of five rats as follows: virgin, pregnant, diabetic virgin, and diabetic pregnant. Diabetes was induced using streptozotocin administration ( $40 \mathrm{mg} / \mathrm{kg}$ i.v.). The rats were lethally anesthetized, and the urethra and vagina were extracted as a unit. Cryostat sections $(6 \mu \mathrm{m}$ thick) were cut and stained with hematoxylin-eosin, and immunohistochemical procedures were performed and subjected to morphological and semi quantitative analysis.

Results. The urethral striated muscle from the diabetic pregnant rats presented with the following variations: thinning and atrophy, disorganization and disruption associated with the colocalization of fast and slow fibers and a steady decrease in the proportion of fast vs slow fibers.

Conclusion. Diabetes and pregnancy impair the urethral striated muscle and alter its fiber type distribution.

\section{Introduction}

Recent studies have established that urinary incontinence (UI) is more prevalent among women with diabetes. ${ }^{1,2}$ In a previous paper, we found a statistically significant association between diabetes mellitus (DM) during pregnancy and UI and pelvic floor muscle dysfunction two years after a Caesarean section (CS). The overall prevalence of gestational UI two years post partum was significantly higher among women with previous GDM (50.8\% vs $44.4 \%$, respectively) than among normoglycemic pregnant women $(31.6 \%$ vs $18.4 \%$, respectively). ${ }^{1}$

The risk factors for pelvic floor muscle dysfunction among these women were related to a high newborn weight and high maternal weight gain during pregnancy because of gestational diabetes mellitus (GDM). Furthermore, the risk factors for UI were indirectly influenced by GDM and pelvic floor muscle dysfunction. This framework confirms an association between GDM and subsequent pelvic floor muscle dysfunction two years after a CS. ${ }^{1}$

The roles of pregnancy and childbirth in determining UI are still controversial. Many hypotheses have attempted to explain the origin of UI during pregnancy, its association with vaginal delivery, and the protective effect of a CS. The observed increase in the concentration of collagen and the decrease in muscle fibers in the urethras of female rats after vaginal delivery may provide insight into the mechanisms involved in the development of UI in women. ${ }^{2}$ However, Barbosa et al., showed that an elective CS was insufficient for preventing UI two years post partum. ${ }^{3}$

$\mathrm{UI}$ is a debilitating disorder caused by a malfunctioning urethral sphincter. ${ }^{4,5}$ Stronger clinical support for a causal relationship between a decreased urethral sphincter thickness and UI has been reported. ${ }^{6}$

Striated fibers are the dominant muscle components of the mid-urethra ${ }^{7}$ and have been classified into two major groups, type I (slowtwitch) and type II (fast-twitch) fibers, based on the presence of myosin heavy chain (MHC) isoforms. Slow-twitch type-I muscle fibers are rich in mitochondria, exhibit a high oxidative capacity, and are resistant to fatigue. Conversely, fast-twitch type II muscle fibers have robust glycolytic metabolism and fatigue easily. ${ }^{8}$ The roles of each fiber type in striated sphincter contraction are controversial and depend on the species studied and the method used to determine the fast and slow fiber types. $^{9}$

Given the high prevalence of UI among women with previous GDM and that striated muscle is one of the two most important tissue types affected by insulin resistance and type 2 diabetes, the purpose of this study was to eval-
Correspondence: Marilza Vieira Cunha Rudge, Departamento de Ginecologia e Obstetrícia Faculdade de Medicina de Botucatu, UNESP, CEP. 18618.000 Botucatu, São Paulo, Brazil.

Tel. +55.14.38116181

E-mail: marilzarudge@ig.com.br

Key words: diabetes, pregnancy, striated muscle fiber, urethra.

Acknowledgements: the authors thank the Research Center in Neurology (University of São Paulo) for technical assistance and Drs. Rodrigo Aquino de Castro, Jorge Milhem Haddad and Suely Kazue Nagahashi Marie. Financial support: FAPESP (Process Number 2008/00989-4).

Contributions: all contributed equally to the manuscript.

Conflict of interest: the authors report no conflicts of interest.

Received for publication: 19 May 2011

Accepted for publication: 11 October 2011.

This work is licensed under a Creative Commons Attribution NonCommercial 3.0 License (CC BYNC 3.0).

(C) Copyright G. Marini et al., 2011

Licensee PAGEPress, Italy

Urogynaecologia 2011; 25:e9

doi:10.4081/uij.2011.eg

uate the urethral striated muscle fiber composition of the urethras of diabetic pregnant rats to understand the influences of diabetes and pregnancy on urethral muscle fibers. Alterations in the two basic types of urethral striated fibers, type I (slow) and type II (fast) fibers, were analyzed in the urethral muscles of pregnant diabetic rats. We hypothesized that diabetes and pregnancy would detrimentally affect the normal function of urethral striated muscle in rats, providing a model for additional studies related to UI.

\section{Materials and Methods}

This study was developed in the Experimental Research Laboratory of the Department of Gynecology and Obstetrics, Botucatu Medical School, UNESP São Paulo, Brazil. Prior to the study, ethical approval was obtained from the Institutional Animal Care and Use Committee on Animal Experimentation at the Botucatu Medical School-UNESP (process number 668).

\section{Animals}

Six-week-old female and nine-week-old male Wistar rats, weighing approximately 180 
g and $220 \mathrm{~g}$, respectively, were allowed to adapt to the laboratory for seven days. The rats were housed in collective cages under controlled temperature $\left(22 \pm 3^{\circ} \mathrm{C}\right)$, light $(12 \mathrm{~h}$ light/dark cycles) and relative humidity $(60 \pm 5 \%)$ conditions. The animals were fed laboratory chow (Purina ${ }^{\circledR}$ ) and tap water ad libitum and cared for in accordance to the principles in the Guide for the Care and Use of Experimental Animals.

The adult female rats were distributed among four groups:

Control group 1: five virgin rats euthanized on a similar period as pregnant group;

Control group 2: five pregnant rats that underwent a CS at the term of pregnancy and were immediately euthanized;

Control group 3: five diabetic virgin rats that were euthanized on a similar period as the pregnant group;

Study group 4: five diabetic pregnant rats that underwent a CS at the term of pregnancy and were immediately euthanized.

\section{Induction of diabetes}

Diabetes was induced using streptozotocin (STZ; SIGMA Chemical Company, St. Louis, M0, USA) seven days prior to mating. A dose of $40 \mathrm{mg} / \mathrm{kg}$ body weight was intravenously administered to produce a permanent and severe diabetic state. The rats' blood glucose levels were measured at the beginning and end of the experimental period using glucose oxidase reagent strips (One-Touch Ultra Johnson \& Johnson ${ }^{\circledR}$, Milpitas, CA, USA). Only the rats with glucose levels greater than $200 \mathrm{mg} / \mathrm{dL}$ were assigned to the diabetic groups. ${ }^{10}$

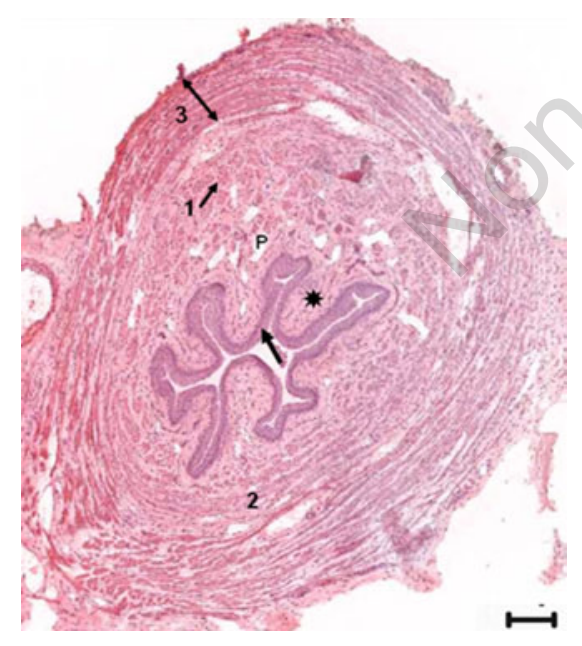

Figure 1. Microphotograph of transverse section of the central part of the urethra in a virgin female rat. Different layers are evident from the lumen to the periphery: epithelium (arrow), lamina propria (*), spongy vascular plexus (P), smooth muscle: longitudinal (1) and circular (2) oriented fibers, and striated muscle (3). H\&E stained. Scale bar, $100 \mu \mathrm{m}$.
The female rats (the pregnant and diabetic pregnant groups) were mated overnight with non-diabetic male rats. The morning when sperm were found in the vaginal smear was designated as gestational day 0 .

On day 21 of pregnancy, the fed rats were weighed to determine the maternal weight gain (final weight - initial weight) and lethally anesthetized with sodium thiopental (3\% Thiopentax ${ }^{\circledR}$ ). The urethra and vagina were extracted as a unit to facilitate their handling. Each unit was immediately placed in a position suitable for transverse sectioning and frozen with liquid nitrogen. The samples were stored at $-80^{\circ} \mathrm{C}$ until sectioning and staining. Cryostat sections $(6 \mu \mathrm{m}$ thick) were cut and stained with hematoxylin-eosin (H\&E) to visualize the nuclei, membranes, cytoplasm and connective tissue. Immunohistochemical procedures were

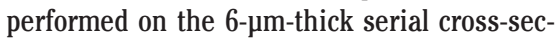
tions to visualize the fast and slow myosin heavy chains. A myosin heavy chain (slow) NCL-MHCs mouse monoclonal antibody (Novocastra; 1:120 dilution) and a myosin heavy chain (fast) NCL-MHCf mouse monoclonal antibody (Novocastra; 1:160 dilution) were used.

\section{Data analysis}

The rat urethra was analyzed using morphological analysis, and a semiquantitative method was used to analyze the immunohistochemical staining of the fast and slow skeletal muscle fibers.

For this analysis, the fast and slow type fibers were considered separately. The immunolocalization intensity was evaluated by averaging the results from two blind and independent readings. The urethral striated muscle was analyzed based on the following parameters: the presence of each type of fiber throughout the circumference of the layer $(++++$ if the layer was complete throughout the circumference to + if the layer was incom- plete); the thickness of the muscle fiber layers (++++ for a thickness of more than five layers to + for a thickness of one muscle fiber layer); the degree that the layers maintained a normal anatomic localization (++++ for a normal anatomic localization to + for a loss of normal anatomic localization).

The scores for the circumferential presence, thickness, and anatomic localization (based on the above criteria) were multiplied for each fiber type. The obtained values of the fast fibers were then divided by those of the slow fibers to establish the fast/slow index.

Convenient transformations (Neperian log) were performed to adjust the offspring weight, maternal weight gain and glycemic data to a symmetrical distribution with a homogeneous variance. Analysis of variance (ANOVA) followed by Tukey's multiple comparison test was used. Statistical significance was considered to be $\mathrm{P}<0.05$. The data were expressed as mean \pm standard error of the mean (SEM).

\section{Results}

\section{Urethral histology}

The transverse sections of the center of the urethra in the virgin group revealed the following various layers from the lumen to the periphery: a stratified squamous epithelium (arrow), a lamina propria (*), a spongy vascular plexus (P), smooth muscle including both longitudinal (1) and circular (2) fibers, and striated muscle (3) (Figure 1).

\section{Morphological and semi quantita- tive analyses of the striated muscle fiber composition of the rat urethra}

\section{The virgin control group}

The H\&E-stained transverse cross-sections

Table 1. Semi-quantitative analysis of slow and fast fibers according the presence of each type of fiber throughout circumference of the layer; thickness of the muscle fiber layer; the degree to which the layers maintained a normal anatomic localization; fast index; slow index; and fast:slow index in each group.

\begin{tabular}{lcccc} 
Groups & Virgin & Pregnant & Diabetic virgin & Diabetic pregnant \\
$\begin{array}{l}\text { Fast throughout } \\
\text { circumference/ } \\
\text { thickness/ }\end{array}$ & $++++/+++/+++$ & $++++/+++/+$ & $+++/+++/+$ & +++++++ \\
normal anatomic & & & & \\
localization & & & & \\
$\begin{array}{l}\text { Slow throughout } \\
\text { circumference/ } \\
\text { thickness/ }\end{array}$ & $++++/++++$ & $++++/+++$ & $+++/++/+$ & $++++/+$ \\
normal anatomic & & & & \\
localization & & & & \\
\hline $\begin{array}{l}\text { Fast index } \\
\text { Slow index }\end{array}$ & 64 & 32 & 12 & 9 \\
\hline Fast:Slow index & $4: 1$ & 8 & 9 & 6 \\
\hline
\end{tabular}


of the striated muscle fiber revealed many layers and compact outer circular layers. The fibers were long, with a similar thickness throughout the circumference (Figure 2A).

Immunohistochemical staining revealed that the striated myofibers predominantly expressed the fast myosin heavy chain isoform. The layer containing the fast fibers was thick, and the fibers were present throughout the outer circular layer (++++) (Figure 2B). The proportion of fast $v$ s slow fibers was 4:1 (Table 1). A thin, inner circular layer of slow, striated muscle fibers was observed $(+)$ with small and thin individual fibers (Figure $2 \mathrm{C}$ ). The image suggested different localization patterns for each type of fiber, with fast fibers occurring in the outermost layer and slow fibers occurring in the innermost layer.

\section{The pregnant control group}

The H\&E-stained transverse cross-sections revealed of the presence of a striated muscle layer that was similar to that of the control group. An increase in the amount of connective tissue separated the fibers from one another. The most important findings in this group were the large interstitial spaces found between the fibers (Figure 3A). Immunohistochemical staining revealed that the distribution of fast $v s$ slow fibers and the proportions of each were similar to those of the virgin group (4:1) (Figure 2 E,F) (Table 1).

\section{The diabetic virgin control group}

H\&E-stained transverse cross-sections revealed that the circular annulus was lost. Additionally, there was fiber thinning and atrophy, and the striated muscle was disrupted. Complete striated muscle layers were scarce (Figure 3B).

Immunohistochemical staining revealed that the specific localization of each type of fiber was lost, with fast and slow fiber colocalization and a decrease in the proportion of fast vs slow fibers to 1.5:1 (Figure $2 \mathrm{H}-\mathrm{I}$ ) (Table 1).

\section{The diabetic pregnant study group}

H\&E-stained transverse cross-sections revealed that the circular annulus was lost. The fiber layers were thin, atrophic, and disorganized, and the striated muscle was disrupted. The findings were similar to those of the pregnant group with respect to the increase in the amount of connective tissue separating the fibers from one another and the large interstitial spaces (Figure 3C).

Immunohistochemical staining revealed the loss of the specific localization of each type of fiber, with fast and slow fiber colocalization and a decrease in the proportion of fast $v s$ slow fibers to 1.5:1 (Figure 4 B,C) (Table 1).

\section{Maternal and perinatal results}

The mean maternal weight gain and offspring weights in the pregnant group showed no statistically significant differences compared to the diabetic pregnant rats (Table 2).

The diabetic virgin and diabetic pregnant

groups presented increased glycemia during pregnancy compared to the virgin and pregnant groups $(\mathrm{P}<0.05)$ (Table 2$)$.

A

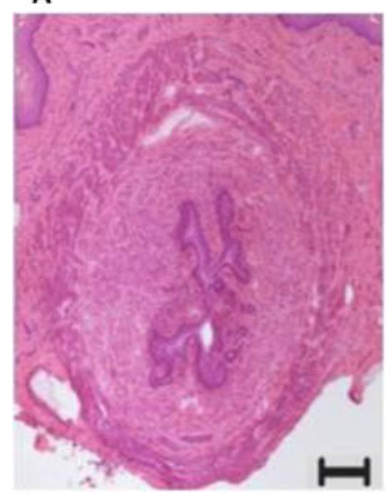

D
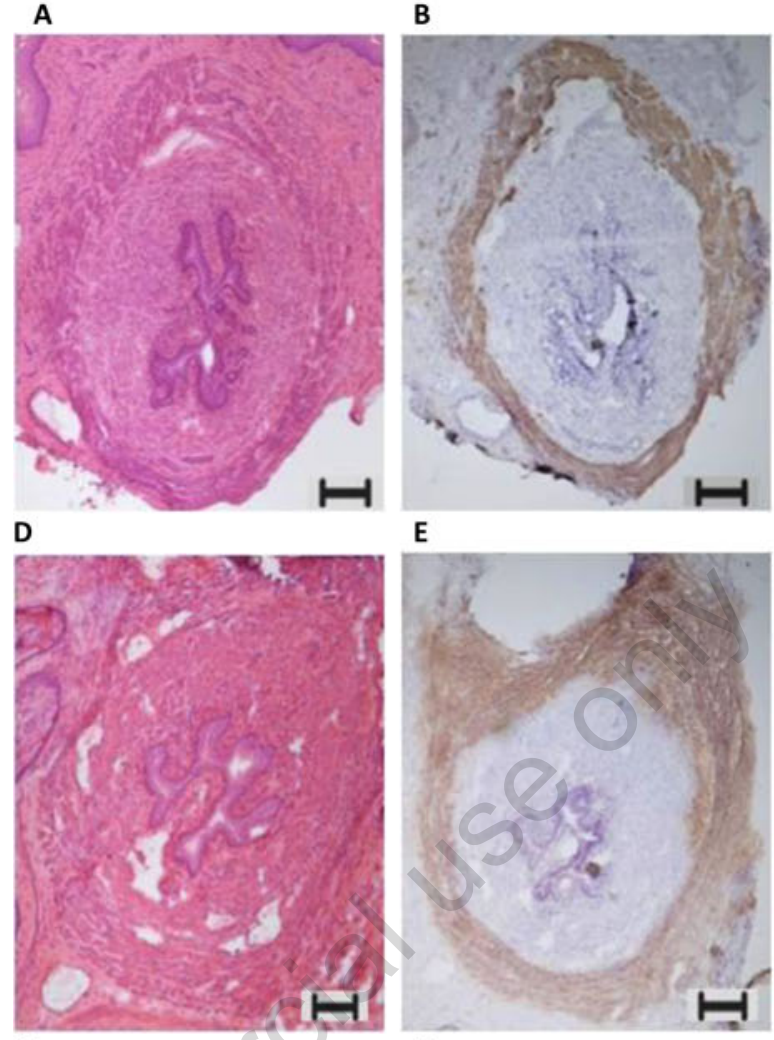

E
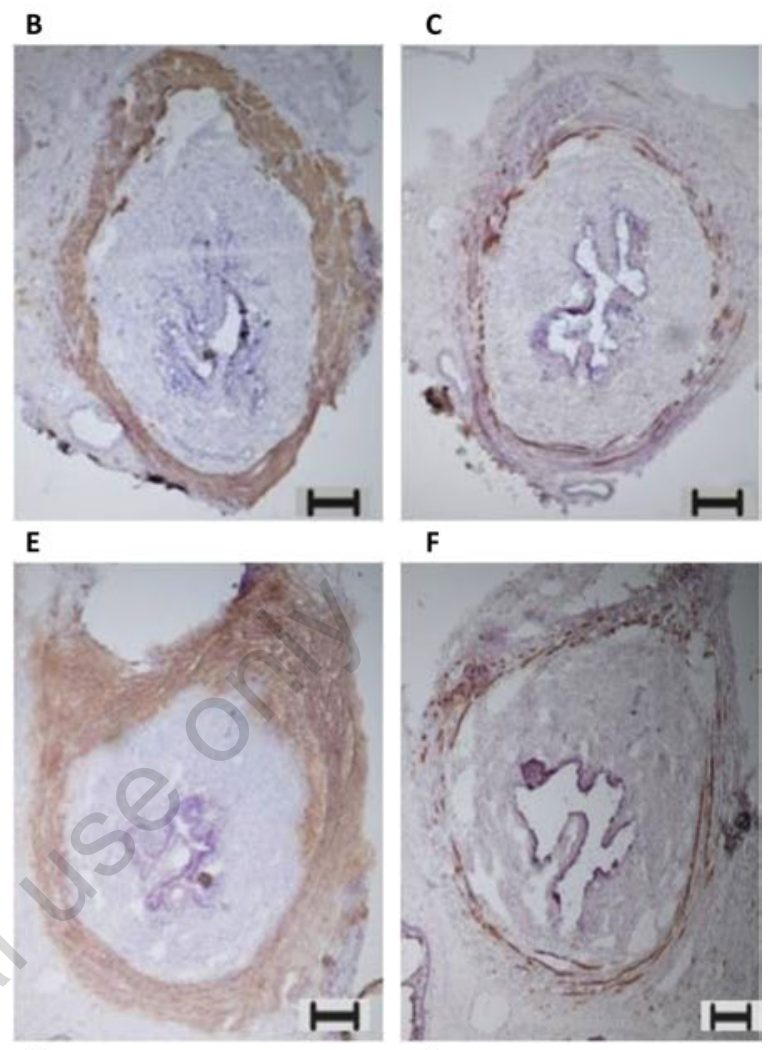

$\mathbf{F}$

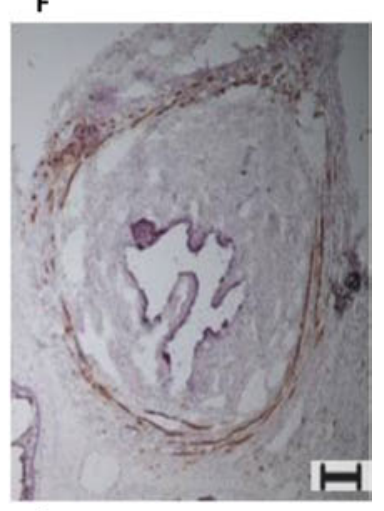

G

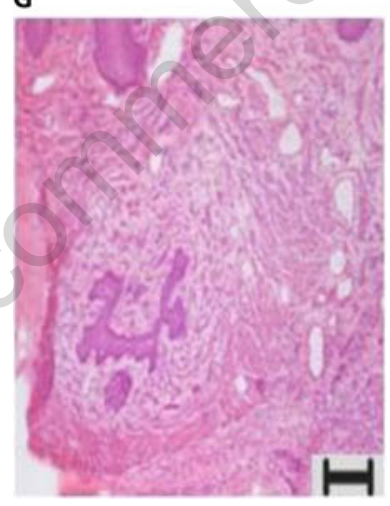

H

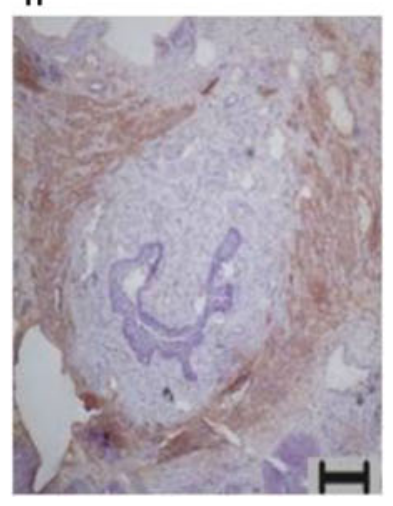

I

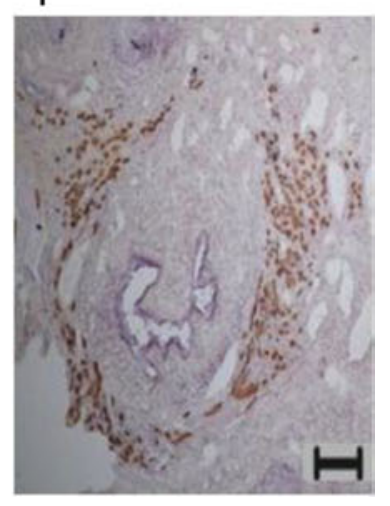

Figure 2. Microphotographs of transverse section of the urethra in virgin group (A, B, $C)$, pregnant group $(D, E, F)$, diabetic virgin group $(G, H, I) . H \& E$ stained $(H \& E)$; immunohistochemical staining to visualize fast (FAST) and slow (SLOW) myosin heavy chain (MHCf, MHCs) in the striated muscle fibers. Scale bar, $100 \mu \mathrm{m}$.

Table 2. Maternal weight gain and offspring weight in pregnant and diabetic pregnant groups. Maternal glycemia (mg/dL) from virgin, pregnant, diabetic virgin and diabetic pregnant groups at beginning and end of the experimental period.

\begin{tabular}{|c|c|c|c|c|}
\hline Groups & Virgin & Pregnant & Diabetic virgin & Diabetic pregnan \\
\hline Maternal weight gain (g) & & $115.4 \pm 16.3$ & & $72.2 \pm 21.5$ \\
\hline Offspring weight (g) & & $82.6 \pm 10.6$ & & $67.2 \pm 14.3$ \\
\hline $\begin{array}{l}\text { Maternal glycemia }(\mathrm{mg} / \mathrm{dL}) \text {, } \\
\text { beginning of experiment }\end{array}$ & $81.0 \pm 4.4$ & $112.8 \pm 4.0$ & $568.4 \pm 38.0^{*}$ & $544.0 \pm 36.8^{*}$ \\
\hline $\begin{array}{l}\text { Maternal glycemia }(\mathrm{mg} / \mathrm{dL}) \text {, } \\
\text { end of experiment }\end{array}$ & $81.6 \pm 5.0$ & $82.6 \pm 7.7$ & $584.8 \pm 33.9 *$ & $497.8 \pm 60.4^{*}$ \\
\hline
\end{tabular}

Values are reported as mean $\pm \mathrm{SEM} ;{ }^{*} \mathrm{P}<0.05$, significant statistically difference compared to virgin and pregnant groups (Tukey's Multiple Comparison Test). 


\section{Discussion}

The goal of this study was to gain a more comprehensive understanding of the striated muscle fiber composition of the urethra of the pregnant diabetic rat and the proportions of the two basic types of urethral striated muscle fibers (type I (slow-twitch) and type II (fasttwitch) fibers). It is important to understand the effects of DM and pregnancy on striated muscle and develop new therapeutic strategies. Human studies are often limited due to ethical concerns, the challenges of obtaining large tissue samples, and the use of strictly managed control groups. To understand how the various risk factors for UI affect the morphological properties of striated muscle, animal models are useful because the experiments are conducted under controlled conditions. ${ }^{11}$

The striated muscle fiber composition of the diabetic pregnant rat urethra is presented along with the importance of considering the experimental conditions and the inclusion of three control groups (virgin, pregnant and diabetic virgin). With this methodology, it was possible to separately analyze the influences of diabetes vs pregnancy. Of particular note, we found that, relative to these three groups, the urethral striated muscles of the diabetic pregnant rats presented the following: thinning, atrophy, disorganization, disruption in the circular annulus associated with the co-localization of fast and slow fibers, and a steady decrease in the proportion of fast vs slow fibers (fast:slow, 1.5:1). The amount of connective tissue separating the fibers from one another (i.e., in the interstitial spaces between the fibers) increased as an effect of pregnancy on urethral muscles. Our results confirm that diabetes per se was implicated in the pathological findings, and pregnancy was only related with respect to the thickness of muscle fiber layer.

Thinning, atrophy, disorganization, and disruption in the circular annulus of striated muscle were extensive damage caused by diabetes. ${ }^{12,13} \mathrm{DM}$ is related to the accumulation of reactive oxygen species, and tissue ischemia can contribute to the myopathic causes of skeletal muscle dysfunction dependently or independently. ${ }^{14-16}$ Previously, the relationship between oxidative stress and diabetes in pregnant rats was confirmed by our group in a report by Damasceno et al. ${ }^{17}$ Increasingly, reports have suggested a potential role for inflammation in the pathogenesis of type 2 DM. This has been supported by the results of both pre-clinical studies and new clinical trials using anti-inflammatory approaches to treat the disease. ${ }^{18}$

By analyzing this data, we were able to explain that extensive damage to striated muscle fibers characterized by reduced skeletal

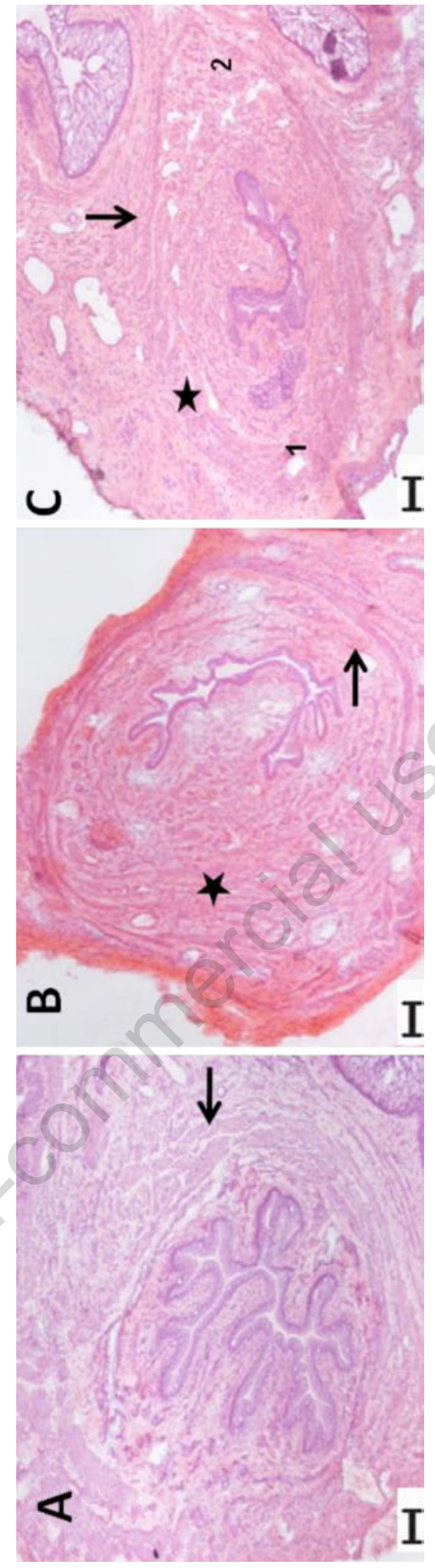

Figure 3. Microphotographs of transverse section of the rat urethra: A - pregnant group: increase in connective tissue separated the fibers from one another (arrow). B - diabetic virgin group: the striated muscle showed thinning and atrophy (arrow), and disruption (*). C - diabetic pregnant group: the striated muscle showed intersticial spaces (1), thinning and atrophy (2), disorganization (arrow), and disruption (*). H\&E stained. Scale bar, $100 \mu \mathrm{m}$.
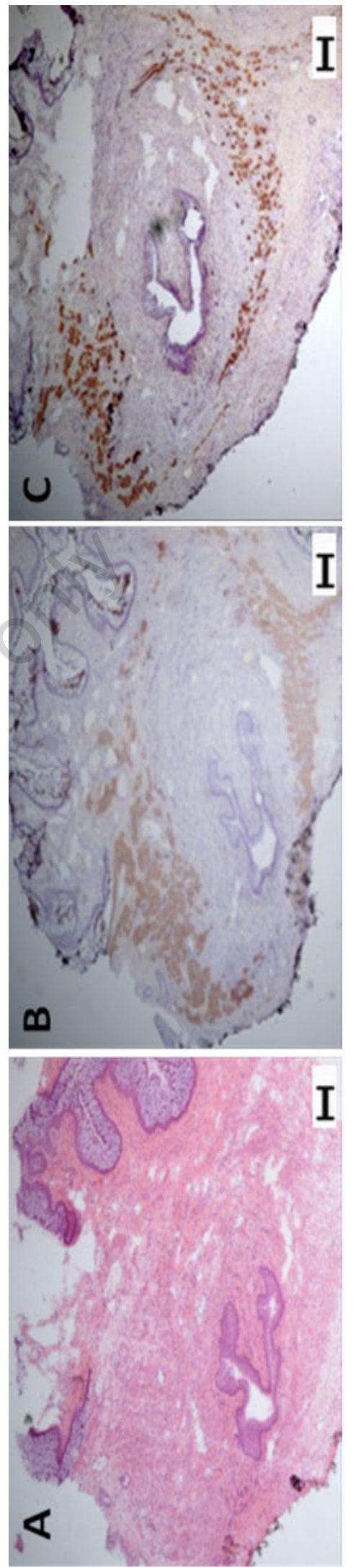

Figure 4. Microphotographs of transverse section of the rat urethra in diabetic pregnant group. H\&E stained (A), immunohistochemical reaction for fast $(B)$ and slow (C) myosin heavy chain (MHCf, MHCs). Scale bar, $100 \mu \mathrm{m}$. 
muscle mass and an altered myofiber composition links diabetes and pregnancy to UI in diabetic pregnant rats. This specific loss of skeletal muscle mass is called diabetic myopathy. ${ }^{19}$ This study confirms previous findings showing that both diabetic myopathy and pregnancy are involved in the pathogenesis of UI.

Differences in the fiber type composition were detected in the urethral striated muscles of the diabetic pregnant rats compared to the control groups. In the studied animals, the expression profiles of the fast vs slow fibers revealed two main differences. First, fast fibers lost their predominance with respect to slow fibers. Second, the fast fibers lost their typical architecture, and the tissue was transformed into a mixture of slow and fast fibers. To the best of our knowledge, these findings are described here for the first time and may be labeled as a diabetic pregnant myopathy. Studies in animal models have shown a strong relationship between muscle fiber types and the development of diabetes. ${ }^{20}$

Skeletal muscle is responsible for movement and is the largest organ for glucose utilization. Our finding of an increased number of type I (slow-type) fibers could be related to the abundant availability of lipids. ${ }^{21}$ Changes in a muscle's fiber composition are often associated with glucose metabolism, diabetes and obesity. ${ }^{22}$ Since muscle is an important site for glucose uptake, reduced muscle mass and changes in a muscle's fiber type composition may directly impair acute glucose utilization. Skeletal muscle can adapt to functional and metabolic demands by remodeling (via fibertype switching) to maintain normal energy balance and nutrient utilization.

Chen et al. ${ }^{8}$ confirmed a higher proportion of type I fibers and the presence of fast-to-slow fiber-type switching, which appears to be dissociated from the expected change in oxidative capacity. Our findings suggest that DM alters the profile of fast vs slow fibers in the urethral striated muscles of diabetic pregnant rats, and eventual fiber-type switching could be present. The nature of the mechanism underlying the altered fiber types in our model requires further investigation.

Because the primary function of the lower urinary tract is the storage and expulsion of urine at the appropriate times, changes in the striated muscle composition may be related to the loss of type-II fibers ${ }^{23}$ or the transformation of most type II fibers into type I fibers..$^{24}$ Given the limitations of this study, its results could represent muscle changes that depend on glucose levels or provide early evidence for tissue inflammation during the pathogenesis of insulin resistance and type $2 \mathrm{DM} .^{25}$

The damage revealed by the morphological studies demonstrates the associated impacts of diabetes and pregnancy on urethral striated muscle fibers, as three of the factors related to altered urethral striated muscle during diabetes and pregnancy (the maternal weight gain, the weight of the offspring and any trauma related to a vaginal delivery) were controlled. However, the results of our study should be interpreted with the awareness of the following limitations: rats are quadrupeds; they have tails with associated musculature; their bladders are abdominal and not pelvic organs. $^{12}$

It is well established that the functional capacity of a muscle is impaired when its fibers are injured. ${ }^{26}$ As the function of a skeletal muscle is determined by its mass and fiber composition, ${ }^{8}$ our results provide evidence that, in a translational study, diabetes and pregnancy injure striated muscles and alter their fast and slow fiber compositions in rats. These data suggest that diabetic pregnant rats may present altered urethral striated muscle contractility, supporting the high prevalence of UI in women with previous GDM two years after a CS. ${ }^{1}$

The importance of this study is the support of the previous clinical hypothesis that diabetes and pregnancy detrimentally affect the normal function of urethral striated muscles in rats, which provides a model for additional studies.

\section{Conclusions}

This study allowed us to describe the morphological changes in muscle mass and the fast $v$ s slow fiber profiles of the urethral striated muscle fibers of diabetic pregnant rats. The urethral striated muscles were found to be thin, atrophic, disorganized, and disrupted. They were associated with the loss of the normal anatomic localization of each fiber type (i.e., the colocalization of fast and slow fibers and the loss of the predominance of the expression of the fast fibers with respect to the slow fibers). The results of this translational study suggest that UI may be attributed, in part, to the changes in urethral striated muscles of diabetic pregnant women.

\section{References}

1. Barbosa AM, Dias A, Marini G, et al. Urinary incontinence and vaginal squeeze pressure two years post-cesarean delivery in primiparous women with previous gestational diabetes mellitus. Clinics (Sao Paulo) 2011;66:1341-6.

2. Rocha MA, Sartori MG, De Jesus Simoes $\mathrm{M}$, et al. The impact of pregnancy and childbirth in the urethra of female rats. Int Urogynecol J Pelvic Floor Dysfunct 2007
Jun;18:645-51.

3. Barbosa A, Rudge M, Rudge C, et al. Does the elective cesarean protect the woman from the occurrence of urinary incontinence and from pelvic floor muscle dysfunction? FIEP Bulletin 2008;78:202-4.

4. Heesakkers JP, Gerretsen RR. Urinary incontinence: sphincter functioning from a urological perspective. Digestion 2004; 69:93-101.

5. Sebastio N, Simonazzi M, Ferri E, et al. Profile of the urethral transmission pressure in patients undergoing in tensionfree vaginal tape. Urogynaecologia Int $\mathbf{J}$ 2000;14:25-30.

6. Morgan DM, Umek W, Guire K, et al. Urethral Sphincter Morphology and Function With and Without Stress Incontinence. J Urol. 2009;182:203-9.

7. Andersson PO, Malmgren A, Uvelius B. Functional responses of different muscle types of the female rat urethra in vitro. Acta Physiol Scand 1990;140:365-72.

8. Chen M, Feng HZ, Gupta D, et al. G(s)alpha deficiency in skeletal muscle leads to reduced muscle mass, fiber-type switching, and glucose intolerance without insulin resistance or deficiency. Am J Physiol Cell Physiol 2009 Apr;296:C930-40.

9. Elbadawy A. Functional anatomy of organs of micturition. Urol Clin North Am 1996; 23:177-210.

10. Calderon IMP, Rudge MVC, Ramos MD, Peraçoli JC. Estudo longitudinal, bioquímico e histoquímico de placentas de ratas diabéticas: relação com a macrossomia e o retardo de crescimento intra-uterino. Rev Bras Ginecol Obstet 1999;21:91-8.

11. Abramowitch SD, Feola A, Jallah Z, Moalli PA. Tissue mechanics, animal models, and pelvic organ prolapse: a review. Eur J Obstet Gynecol Reprod Biol 2009;144: S146-58.

12. Kim JH, Xiao H, Liu G, et al. Diabetes slows the recovery from urinary incontinence due to simulated childbirth in female rats. Am J Physiol Regul Integr Comp Physiol 2007;293:950-5.

13. Gasbarro G, Lin DL, Vurbic D, et al. Voiding function in obese and type 2 diabetic female rats. Am J Physiol Renal Physiol 2010;298:F72-7.

14. Kim RJ, Kerns JM, Liu S, et al. Striated muscle and nerve fascicle distribution in the female rat urethral sphincter. Anat Rec (Hoboken) 2007;290:145-54.

15. Beshay E, Carrier S. Oxidative stress plays a role in diabetes-induced bladder dysfunction in a rat model. Urology 2004; 64:1062-7.

16. De Angelis KL, Cestari IA, Barp J, et al. Oxidative stress in the latissimus dorsi muscle of diabetic rats. Braz J Med Biol Res 2000;33:1363-8. 
17. Damasceno DC, Volpato GT, Calderon IMP, Rudge MVC. Oxidative stress and diabetes in pregnant rats. Animal Reprod Science 2002;72:235-44.

18. Donath MY, Shoelson SE. Type 2 diabetes as an inflammatory disease. Nat Rev Immunol 2011;11:98-107.

19. Krause MP, Riddell MC, Gordon CS, et al. Diabetic myopathy differs between Ins2Akita+/- and streptozotocin-induced Type 1 diabetic models. J Appl Physiol 2009;106:1650-9.

20. Schuler M, Ali F, Chambon C, et al. PGClalpha expression is controlled in skeletal muscles by PPARbeta, whose ablation results in fiber-type switching, obesity, and type 2 diabetes. Cell Metab
2006;4:407-14.

21. de Wilde J, Mohren R, van den Berg S, et al. Short-term high fat-feeding results in morphological and metabolic adaptations in the skeletal muscle of C57BL/6J mice. Physiol Genomics 2008;32:360-9.

22. Oberbach A, Bossenz Y, Lehmann S, et al. Altered fiber distribution and fiber-specific glycolytic and oxidative enzyme activity in skeletal muscle of patients with type 2 diabetes. Diabetes Care 2006;29:895-900.

23. Pandit M, Delancey JOL, Ashton-Miller, et al. Quantification of intramuscular nerves within the female striated urogenital sphincter muscle. Obstet Gynecol 2000;95: 797-800.

24. Fujimoto S, Watanabe J, Ogawa R,
Kanamura S. Age related changes in fibre number fibre size, fibre type composition and adenosine triphosphatase activity in rat soleus muscle. Ann Anat 1994;176:42935 .

25. Hotamisligil GS, Shargill NS, Spiegelman BM. Adipose expression of tumor necrosis factor-alpha: direct role in obesity-linked insulin resistance. Science 1993;259:8791.

27. Gregorevic P, Plant DR, Stupka N, Lynch GS. Changes in contractile activation characteristics of rat fast and slow skeletal muscle fibres during regeneration. J Physiol 2004;558:549-60. 